\title{
Low Power Wireless Sensor Networks Algorithm: EASRP
}

\author{
Smt. Sheetalrani R Kawale ${ }^{1}$, Prof. G. Mahadevan ${ }^{2}$,Samartha G. ${ }^{3}$ \\ ${ }^{1}$. Assistant Professor, Dept. of Comp. Sci., AWU, Vijayapura, Karnataka, India \\ 2. Professor, Dept. of Comp. Sci., AMC Engineering collage, Bangalore, Karnataka, India \\ 3. Samartha G, Dept. of Comp.Sci., Acharya Engineering collage, Bangalore, Karnataka, India
}

\begin{abstract}
Wireless Sensor Networks comprises of a huge number of communicating devices called as, sensor node. These nodes are powered by a limited battery source. Moreover, the sensor nodes will be instigated in harsh and hostile environment, after which, battery replacement become very difficult. Hence energy conservation and power saving is very crucial. The LEACH provides good energy conservation, but there is still scope for some improvement, which is addressed by the proposed ENERGY AWARE SECURE ROUTING PROTOCOL (EASRP) scheme.
\end{abstract}

Key Words: Wireless Sensor Networks, Clustering, AES, DES, Clusters, Network Lifetime, LEACH, Encryption.

\section{Introduction}

A Wireless Sensor Networks comprises of various wireless devices which observe and sense variations in physical or ecological conditions, for example, temperature, moisture, humidity, atmospheric pressure, or toxins, etc., at various territories. Such Networks are required to be instigated in a wide range of environments for business, industrial and military applications, for example, medical purposes, object tracking, surveillance, atmosphere and climate monitoring, and acoustic information, etc. The main drawbacks of wireless sensor networks are the storage capacity, energy, memory and processing. network topologies like Star, Mesh, Bus,etc.

\section{Existing System}

The recent developments in communication technology has empowered the advancement of costeffective, energy-aware, multifunctional network devices for wireless networking applications. The blend of these variables has enhanced the capability of using a sensor network systems with huge amount of sensor nodes, which helps in data accumulation, aggregation, handling and transmission, under various environmental conditions. A sensor system is made out of an extensive number of sensor nodes which comprise of detecting, processing and transmitting the data.

\section{Problem Identification}

Wireless sensor network are used in a wide range of application and purposes. To ensure their proper working, the network needs to be more accurate and routing protocols must be efficient. In order to design new protocols, certain challenges must be considered [3], they are given below.

$>$ Energy Constraints: it is the most significant drawback of the wireless sensor network. Since the nodes are powered by batteries, the available energy is always limited. A depleted battery is also hard to replace or recharge. Once the battery is completely discharged, the node will switch off, which creates a break in the network. Therefore, the lifetime of the network is adversely affected. Subsequently the energy utilization is principle concern for any routing protocol. Low memory and low processing power is another limitation of nodes. Therefore, it is essential for any routing algorithm to be efficient, light-weight and should consume less energy.

$>$ Security: Security is another crucial parameter for wireless sensor networks. Increasing the security will increase the computations. Hence, there is always a trade-offs between security and bandwidth of the network. As the wireless sensor networks are deployed over insecure and harsh environment, data security is very important. Most of the routing protocols does not provide viable security measure which is highly risky. Hence a secure routing protocol with energy-aware mechanism is needed.

$>$ Quality of Service: The data in wireless sensor networks are very critical and carry important information. They must be delivered on time without any information leakage or tampering. The QoS parameter ensure that the message will be delivered within restricted time and not causing any damage to the data.

$>$ Fault-Tolerance: In any harsh working domain, a sensor node may be exposed several adversaries, which may lead to node failure. A failed node hinders network communication and finally degrade the network lifetime. Therefore, the routing protocols must be effective enough to prevent the node from failures. This will not only enhance the network lifetime, but also increases energy conservation. 


\section{Energy Aware Secure Routing Protocol (Easrp)}

The LEACH has several drawbacks, which reduce the efficiency and performance of the network. Such as the sensor nodes are obliged to send their residual energy to cluster heads or base station at each round. This substantially increases the energy consumption and the resources such as processor and memory are wasted. The additional data transfer also increases channel traffic, and available bandwidth will decrease. It will eventually lead to low lifetime of the network, Cluster heads positions and numbers are not considered and the communication in WSN is not entirely secured.

The measures taken to overcome the challenges of LEACH are given below. These parameters also form a foundation for the proposed algorithm, they are,

1. Formation of clusters

2. Selecting suitable cluster heads

3. Establishing a secure communication channel between nodes and cluster heads.

4. Providing security to data and transmission channel.

5. Hybrid cryptography system is used to provide security.

6. The system uses AES and DES algorithms for encryption.

With this proposed work, a new energy aware secure routing protocol(EASRP) is proposed as an enhancement to traditional LEACH.

\section{System Design Description}

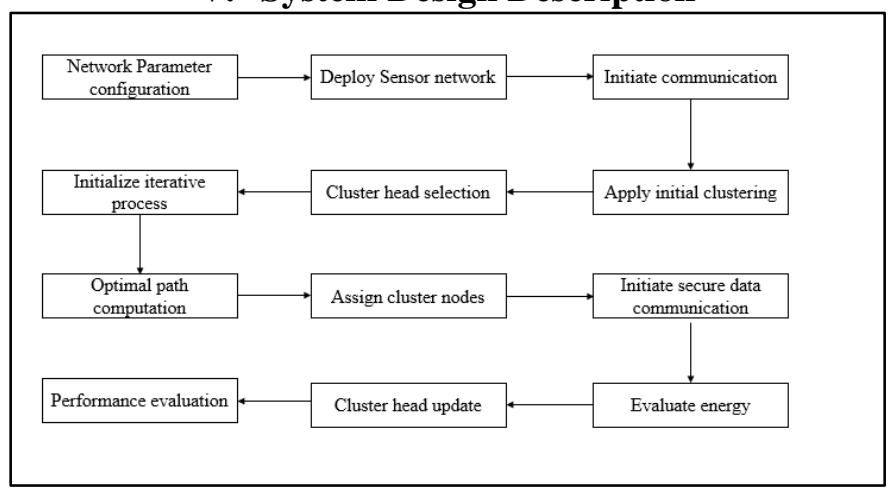

Fig.1 System Architecture

Figure shows the system architecture diagram for the routing algorithm.

Step 1: Define Network parameters

Step 2: Deploy N number of Nodes

Step 3: Initialize network communication

Step 4: Apply initial clustering technique

Step 5: Cluster head Selection (Higher Energy Node will be selected as head)

Step 6: Do

// here starts iterative updation of energy, clusters, cluster head and optimal distance for communication

Step 7: for each cluster head $(\mathrm{CH})$

Step 7 : for each node $(\mathrm{n})$

(a) Distance computation for optimal path selection $(d)$

(b) Assigning nodes to the corresponding closest cluster $c \rightarrow C$

(c) Optimal path computation using various hop counts based on the distance (Path $\rightarrow$ Min $_{\text {Distance }}$ )

(d) Initialize data transmission from source node to destination node.

(e) Apply hybrid data encryption during transmission

(f) Transmit through optimal hop counts i.e. start from source node and deliver to destination by passing through optimal nodes

(g) Evaluate energy per iteration

(h) Dead and alive node computation

(i) Update cluster and cluster head based on energy and distance

(j) Repeat until total number of iterations achieved

(k) End

Step 7: performance evaluation in terms of packet delivery, throughput, energy etc. 


\section{Result And Conclusion}

6.1 Dead Node Performance analysis between EASRP and LEACH

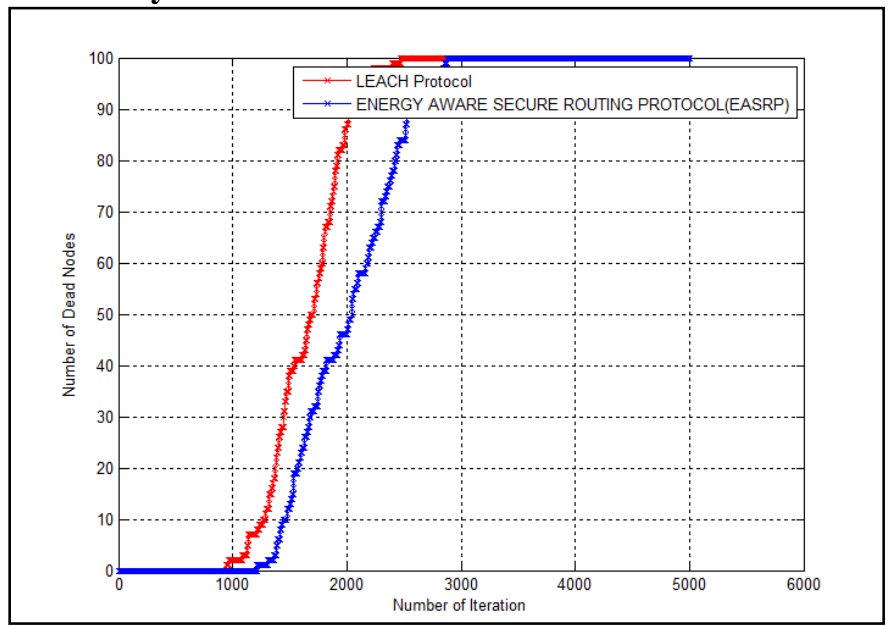

Chart1: Dead Node Performance analysis

6.2 Alive Performance analysis between EASRP and LEACH

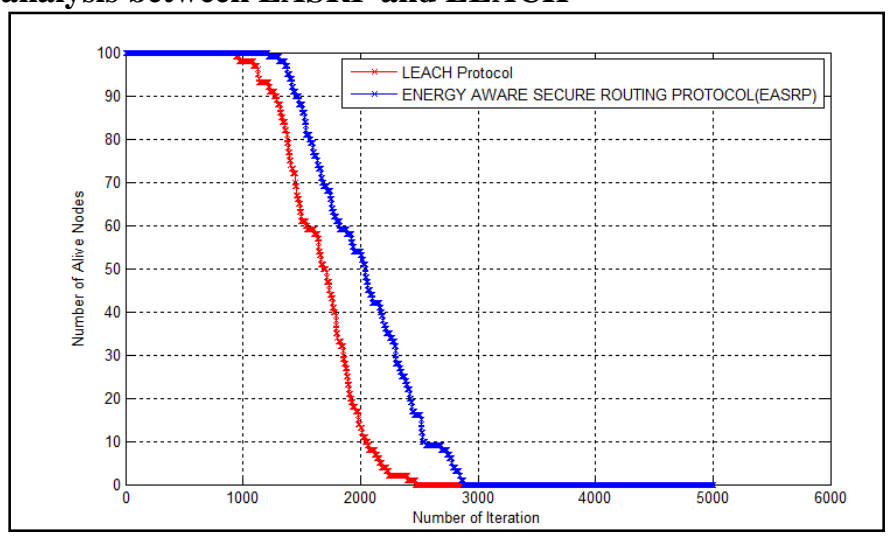

Chart2: Alive Node Performance analysis

6.3 Packet delivery performance between EASRP and LEACH

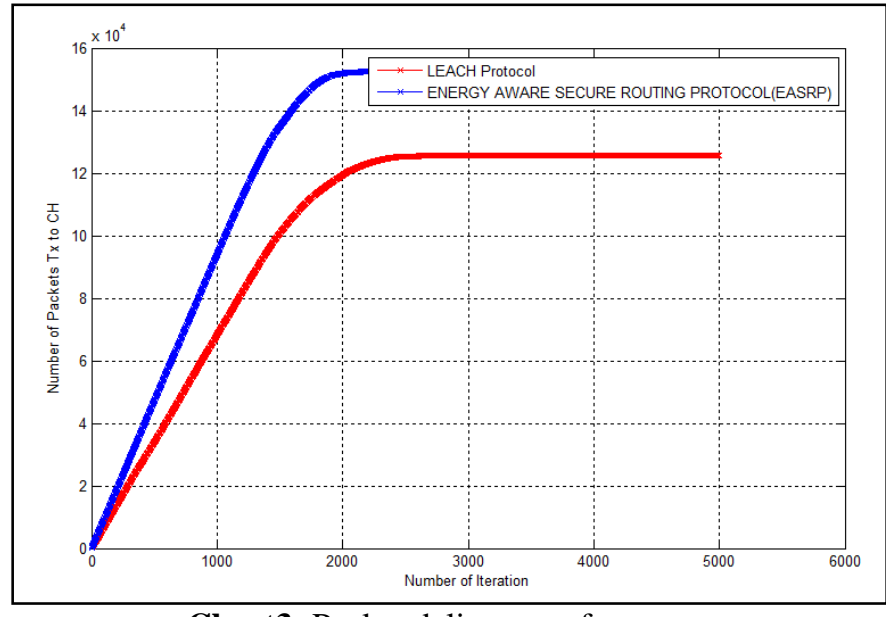

Chart3: Packet delivery performance 


\subsection{End-to-end throughput (QOS) between EASRP and LEACH}

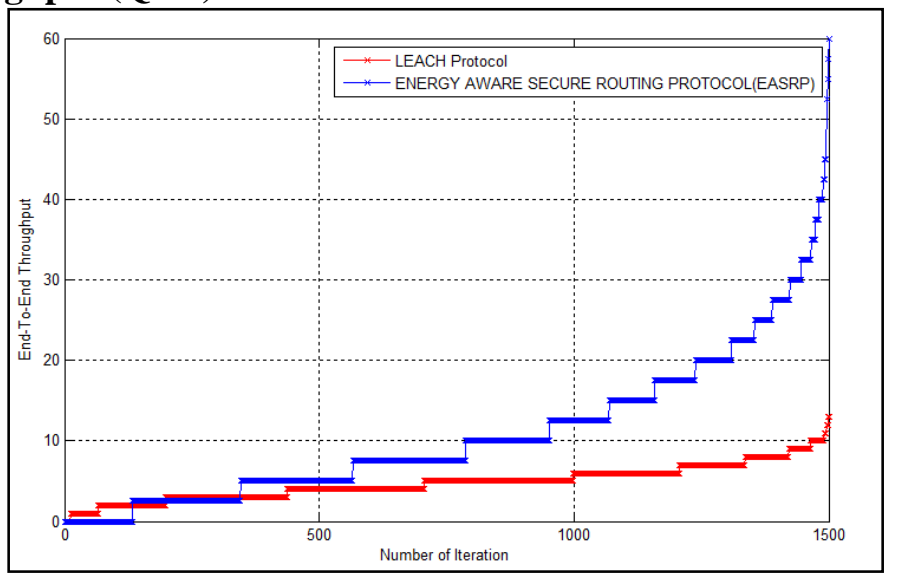

Chart4: End-to-end throughput (QOS)

\subsection{Average Energy-consumption performance between EASRP and LEACH}

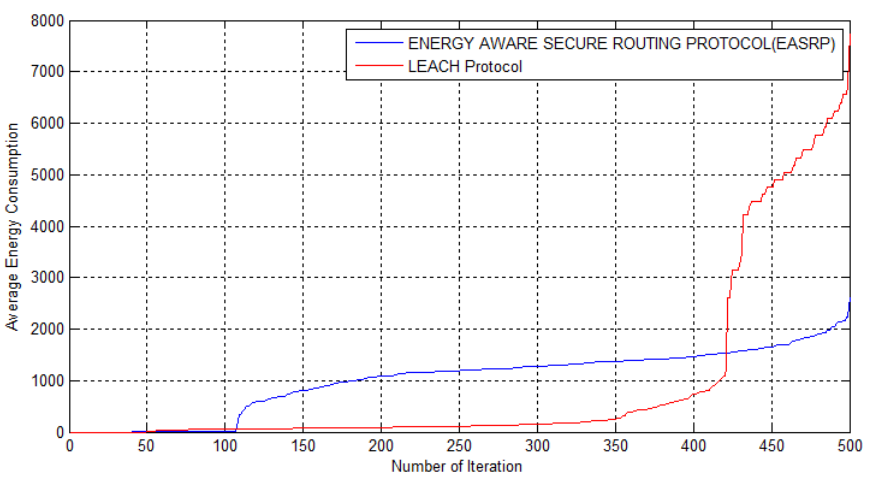

Chart5: Average Energy-consumption performance

\section{Conclusion}

This experimental study shows that proposed approach consumes less average energy when compared with conventional LEACH protocol. Average energy consumption using LEACH is 848.247 (joules) whereas proposed approach consumes 482.31 for complete simulation.

\section{Acknowledgement}

I would like to acknowledge my guide Prof. G. Mahadavan for guiding me and for his kind support.

\section{References}

[1]. W. Su Y. Sankarasubramaniam E. Cayirci Akyildiz, I.F. A survey on sensor networks. IEEE Communications Magazine, pages $102\{114,2002$.

[2]. Kumar.S.P. Chee-Yee Chong. Sensor networks: Evolution, opportunities, and challenges. Proc IEEE, August 2003.

[3]. Ismail H. Kasimoglui Ian .F. Akyildiz. Wireless sensor and actor :research challenges. (Elsevier) Journal, 2(38):351 \{367, 2004.

[4]. Jonathan Jen-Rong Chen Prasan Kumar Sahoo and Ping-Tai Sun. E \pm cient security mechanisms for the distributed wireless sensor networks. Proceedings of the IEEE Third International Conference on Information Technology and Applications (ICITA'05), pages $0\{7695\{2316\{1,2005$.

[5]. Sajid Hussain and Abdul W. Matin Jodrey. Energy e \pm cient hierarchical cluster-based routing for wireless sensor networks. Technical Report - TR-2005-011,2005.073720m@acadiau.ca.

[6]. C. Poellabauer and W. Dargie, Fundamentals of Wireless Sensor Networks : Theory and Practice, Wiley, 2010.

[7]. S. Madden, M.J. Franklin, J.M. Hellerstein, and W. Tag Hong. "A Tiny Aggregation Service for Ad-Hoc Sensor Networks". SIGOPS Opemting System Review 96, SI (December 2002), pp. 131-146.

[8]. B. Liang, and Z.J. Hass. "Virtual Backbone Generation and Maintenance in Ad Hoc Network Mobility Management". In Proc. of the Nineteenth Annual IEEE Conference on

[9]. M. Yarvis, N. Kushalnagar, H. Singh, A. Rangarajan, Y. Liu, and S. Singh. "Exploiting Heterogeneity in Sensor Networks". In Proc. IEEE INFOCOM"e 05 , vol. 2, Miami, FL, Mar. 2005, pp. 878-890.

[10]. H. Gharavi and K. Ban. "Multihop Sensor Network Design for Wide-band Communications". In Proc. of the IEEE, vol. 91, no. 8, Aug. 2003, pp. 1221-1234. [32] T. Hou and T. Sai. "An Access-based Clustering Protocol for Multihop Wireless Ad hoc Networks". In IEEE Journal on Selected Areas in Communications, vol. 19, no. 7, July 2001, pp. 1201-1210. [11] A. Chakraborti, 
A. Sabharwal, and B. Aazhang. "Multi-hop Communication is Order-optimal for Homogeneous Sensor Networks". In Proceeding of the 3rd International Symposium on Information Processing in Sensor Networks, Berkeley, CA, Apr. 2004, pp. 178- 185.

[11]. J. Pan, Y. Hou, L. Cai, Y. Shi, and S. Shen. "Topology Control for Wireless Sensor Networks". In Proceeding of the 9th Annual International Conference on Mobile Computing and Networking, San Diego, CA, Sept. 2003, pp. 286-299.

[12]. S. Bandopadhya and E. Coyle. "An Energy Efficient Hierarchical Clustering Algorithm for Wireless Sensor Networks". In Proceeding of IEEE INFOCOM 2003, vol. 3, San Frabcisco, CA, Apr. 2003, pp. 1713-1723.

[13]. S. Lindsey, C. S. Raghavendra and K. Sivalingam. "Data Gathering in Sensor Networks using the Int. J. of Advanced Networking and Applications". vol. 2, issue: 02, pp. 570-580 (2010). Energy Delay Metric, in Proc. of the IPDPS Workshop on Issues in Wireless Networks and Mobile Computing, San Francisco, CA, April 2001.

[14]. V. Mhatre and C. Rosenberg. "Homogeneous vs heterogeneous clustered sensor networks: a comparative study". Communications, 2004 IEEE International Conference, 2004.

[15]. W.R. Heinzelman. "Application-Specific Protocol Architecture for Wireless Networks". Ph.D Thesis, Massachusetts Institute of Technology, June 2000.

[16]. J. Al-Karaki, and A. Kamal. "Routing Techniques in Wireless Sensor Networks: A Survey". IEEE Wireless Communications, vol. 11, no. 6, Dec. 2004, pp. 6-28.

[17]. Basim Alhadidi, Noor Shahid, Singh Amardeep, "Smart Object Tracking System Using MATLAB", published in International Journal of Computational Intelligence Research (IJCIR), Vol. 8, issue 3, 2012.

[18]. S. Madden, M.J. Franklin, J.M. Hellerstein, and W. Tag Hong. "A Tiny Aggregation Service for Ad-Hoc Sensor Networks". SIGOPS Opemting System Review 96, SI (December 2002), pp. 131-146. [5] G.J. Pottle, and W.J. Kaiser. "Embedding the Internet: Wireless Integrated Network Sensor". Communications of the ACM, vol. 43, no. 5, [May 2000], pp. 51 - 58.

[19]. W.R. Heinzelman, A. Chandrakasan, and H. Balakrishnan. "Energy-efficient Communication Protocol for Wireless Microsensor Networks". in IEEE Computer Society Proceedings of the Thirty Third Hawaii International Conference on System Sciences (HICSS '00), Washington, DC, USA, Jan. 2000, vol. 8, pp. 8020.

[20]. W.R. Heinzelman, A. Chandrakasan, and H. Balakrishnan. "An Application-Specific Protocol Architecture for Wireless Microsensor Networks". In IEEE Tmnsactions on Wireless Communications, (October 2002), vol. 1(4), pp. $660-670$.

[21]. F. Xiangning and S. Yulin, "Improvement on LEACH Protocol of Wireless Sensor Network," in International Conference on Sensor Technologies and Applications, 2007.

[22]. K. Whitehouse, "The design of calamari: an ad-hoc localization system for sensor networks," Master Thesis, University of California, 2003.

[23]. L. Tang and S. Liu, "Improvement on LEACH Routing Algorithm for Wireless Sensor Networks," International Conference on Internet Computing and Information Services, pp. 199-202, 2011.

[24]. "Incorporating Concepts of Nanotechonology Services and Computing For Smart Classrooms" Research Paper Research Link 100, Vol -XI (5) July 2012, Page No. 26-29 RNI No. MPHIN- 2002-7041, ISSN No.-0973-1628

[25]. “ Enhancing Energy Efficiency in WSN using Potential Concepts" International conference on Advances in Computer and Electrical Engineering (ICACEE’2012) Nov. 17-18, 2012 manila (Philippines) ISBN: 978-93-82242-11-6 Page No. 118-122.

[26]. "Enhancing Energy Efficiency in WSN using Energy Potential And Energy Balancing Concepts" International Journal of Innovative Research in Information Security (IJIRIS) volume 1, Issue 2, August 2014 ISSA*/2349-7009(Online) ISSN /2349-7017 (Print) Page no. 15-17.

[27]. "RECR Routing Algorithm In Wireless Sensor Network" International journal Of Emerging Technology \& Research(IJETR) Volume 1, Issue 6, Sep-Oct, 2014 ISSN(E):2347-5900 ISSN (P):2347-6079 Page no. 89-91.

\section{BIOGRAPHIES}

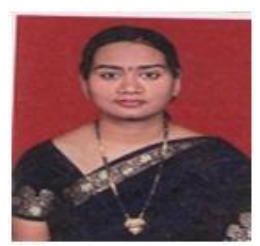

1. Smt. Sheetalrani R. kawale is serving as an Assistant Professor in the Department of Computer Science at the Karnataka State Women's University, Vijayapura. She has 11 yrs of teaching experience and pursuing $\mathrm{PhD}$ in the computer science field. Her area of interest is in networking.

2. Prof. G. Mahadevan is serving as a Professor in the Department of Computer Science and Engg at AMC Engg College Bangalore, India and has published many national and international papers. He has guided more than $15 \mathrm{Ph} . \mathrm{D}$ students and currently 8 students are doing Ph.D under his guide-ship.

3. Samartha G., MCA, Archarya Engineering collage, Bangalore, Karnataka. 